DOI: 10.21915 /BIMAS.2018405

\title{
SYMMETRIC AND ALTERNATING POWERS OF WEIL REPRESENTATIONS OF FINITE SYMPLECTIC GROUPS
}

\author{
ROBERT M. GURALNICK ${ }^{1, a}$, KAY MAGAARD ${ }^{2, b}$ AND PHAM HUU TIEP TI $^{3, c}$
}

\author{
Dedicated to Robert Griess
}

\footnotetext{
${ }^{1}$ Department of Mathematics, University of Southern California, Los Angeles, CA 90089-2532, USA.

${ }^{a}$ E-mail: guralnic@usc.edu

${ }^{2}$ School of Mathematics, University of Birmingham, Edgbaston, Birmingham, B15 2TT, UK.

${ }^{b}$ E-mail: kymgrd@yahoo.com

${ }^{3}$ Department of Mathematics, Rutgers University, Piscataway, NJ 08854-8019, USA.

${ }^{c}$ E-mail: tiep@math.rutgers.edu
}

\begin{abstract}
Motivated by an earlier result of N. Katz, we establish all possible equalities between symmetric squares, alternating squares, and tensor products of complex irreducible Weil characters of finite symplectic groups in odd characteristic. We also construct an infinite series of examples of irreducible symmetric cubes and alternating cubes of complex representations of finite groups.
\end{abstract}

\section{Introduction}

The so-called Weil representations were introduced by A. Weil [30] for classical groups over local fields. Weil mentioned that the finite field case may be considered analogously. This was developed in detail by R. E. Howe [12] and P. Gérardin [8], for characteristic zero representations. The same complex representations were introduced independently by I. M. Isaacs [13] and H. N. Ward [29] for finite symplectic groups $\operatorname{Sp}_{2 n}(q)$ with $q$ odd, and by

Received January 26, 2017.

AMS Subject Classification: 20C33, 20C20.

Key words and phrases: Finite symplectic groups, Weil representations.

The authors are grateful to Nick Katz for bringing the problem to their attention. They are also grateful to the referee for careful reading and helpful comments on the paper.

The first and the third authors gratefully acknowledge the support of the NSF (grants DMS1600056, DMS-1201374, and DMS-1665014). 
G. M. Seitz [22] for finite unitary groups. (These representations for $\operatorname{Sp}_{2 n}(p)$ were also constructed in [3].) Weil representations attract much attention because of their many interesting features, cf. for instance [4], [5], [9], [10], [20], [21], [24], [25], [31].

The construction of Weil representations may be found in [12], [8], [22], etc. Let us recall this construction in the case of $G=\operatorname{Sp}_{2 n}(q)$, where $q$ is a power of an odd prime $p>2$ and $n \geq 1$. Let $V=\mathbb{F}_{q}^{2 n}$ be endowed with a non-degenerate symplectic form $(\cdot, \cdot): V \times V \rightarrow \mathbb{F}_{q}$. Then one checks that $Q:=\left\{(\alpha, v) \mid \alpha \in \mathbb{F}_{q}, v \in V\right\}$, considered with the operation

$$
(\alpha, v) \cdot(\beta, w)=(\alpha+\beta+(v, w), v+w)
$$

is a $p$-group (of special type), with

$$
\mathbf{Z}(Q)=[Q, Q]=\Phi(Q)=\left\{(\alpha, 0) \mid \alpha \in \mathbb{F}_{q}\right\}
$$

being elementary abelian of order $q$. Then $G=\operatorname{Sp}(V)$ acts on $Q$ as outer automorphisms that act trivially on $\mathbf{Z}(Q)$.

Fix a nontrivial linear character $\psi$ of the additive group $\left(\mathbb{F}_{q},+\right)$. Then $\psi$ can be naturally viewed as a character of $\mathbf{Z}(Q)$. One can show, cf. [10, §13], that there is a unique (up to equivalence) complex irreducible representation $\Phi$ of $Q$ of degree $q^{n}$ that affords the character $q^{n} \psi$ of $\mathbf{Z}(Q)$. For any $g \in G$, the action of $g$ on $Q$ fixes $\psi$ and so sends $\Phi$ to an equivalent representation $\Lambda(g) \Phi \Lambda(g)^{-1}$ for some $\Lambda(g) \in \mathrm{GL}_{q^{n}}(\mathbb{C})$. The map $g \mapsto \Lambda(g)$ is in fact a projective representation of $G$ and can be lifted to a linear representation $\Omega$, see $[10, \S 13]$, which is called a reducible Weil representation of $G$, with character $\omega=\omega_{n}$. The latter character can be decomposed into the sum $\eta+\xi$ of two (irreducible) Weil characters, which correspond to the two eigenspaces of $\Omega(\mathbf{j})$, where $\mathbf{Z}(G)=\langle\mathbf{j}\rangle \cong C_{2}$. Here, $\eta$ has degree $\left(q^{n}-1\right) / 2$ and $\xi$ has degree $\left(q^{n}+1\right) / 2$.

The character $\omega$ remains unchanged in the above construction if one replaces $\psi$ by another character $\psi_{a}: x \mapsto \psi(a x)$ as long as $a \in \mathbb{F}_{q}^{\times 2}$. If, however, we replace $\psi$ by $\psi^{*}: x \mapsto \psi(a x)$ for some $a \in \mathbb{F}_{q}^{\times} \backslash \mathbb{F}_{q}^{\times 2}$, then we get a new character $\omega^{*}=\eta^{*}+\xi^{*}$, which is the sum of another pair of Weil characters, $\eta^{*}$ of degree $\left(q^{n}-1\right) / 2$ and $\xi^{*}$ of degree $\left(q^{n}+1\right) / 2$. It is also 
convenient to consider $H=\operatorname{CSp}(V)$, the group of conformal transformations of the symplectic space $V$ :

$$
H=\left\{f \in \mathrm{GL}(V) \mid \exists \kappa(f) \in \mathbb{F}_{q}^{\times},(f(u), f(v))=\kappa(f)(u, v), \forall u, v \in V .\right\}
$$

Then a conjugation by $f \in H$ with $\kappa(f) \in \mathbb{F}_{q}^{\times} \backslash \mathbb{F}_{q}^{\times 2}$ fuses $\omega$ with $\omega^{*}, \eta$ with $\eta^{*}$, and $\xi$ with $\xi^{*}$, see [10, Lemma 13.4].

In the course of his study of finite monodromy groups on $\mathbb{A}^{1}$, Nick Katz [14] noted that, when $n=1$ the alternating square $\wedge^{2}(\xi)$ coincides with either $\operatorname{Sym}^{2}(\eta)$ or $\operatorname{Sym}^{2}\left(\eta^{*}\right)$. The goal of this paper is to show that in fact this phenomenon, as well as several other interesting equalities between symmetric square, alternating squares, and tensor products, of Weil representations indeed occur for all $n$.

Theorem 1.1. Let $q$ be an odd prime power, $n$ any positive integer, and let $\{\eta, \xi\},\left\{\eta^{*}, \xi^{*}\right\}$ be the two pairs of complex Weil characters of $\operatorname{Sp}_{2 n}(q)$ defined above. Then the following statements hold.

(i) $\xi \eta=\xi^{*} \eta^{*}$. However, $\xi \eta^{*} \neq \xi^{*} \eta, \xi^{2} \neq\left(\xi^{*}\right)^{2}$, and $\eta^{2} \neq\left(\eta^{*}\right)^{2}$.

(ii) Suppose $q \equiv \pm 1(\bmod 8)$. Then

$$
\operatorname{Sym}^{2}(\eta)=\operatorname{Sym}^{2}\left(\eta^{*}\right)=\wedge^{2}(\xi)=\wedge^{2}\left(\xi^{*}\right) .
$$

However, $\operatorname{Sym}^{2}(\xi) \neq \operatorname{Sym}^{2}\left(\xi^{*}\right), \wedge^{2}(\eta) \neq \wedge^{2}\left(\eta^{*}\right)$.

(iii) Suppose $q \equiv \pm 3(\bmod 8)$. Then

$$
\begin{aligned}
\operatorname{Sym}^{2}(\eta) & =\wedge^{2}\left(\xi^{*}\right), \operatorname{Sym}^{2}\left(\eta^{*}\right)=\wedge^{2}(\xi), \operatorname{Sym}^{2}(\xi)=\operatorname{Sym}^{2}\left(\xi^{*}\right) \\
\wedge^{2}(\eta) & =\wedge^{2}\left(\eta^{*}\right) .
\end{aligned}
$$

However, $\operatorname{Sym}^{2}(\eta) \neq \operatorname{Sym}^{2}\left(\eta^{*}\right)$ and $\wedge^{2}(\xi) \neq \wedge^{2}\left(\xi^{*}\right)$.

Let $\epsilon:=(-1)^{(q-1) / 2}$. Our proof of Theorem 1.1 relies on the following result which is of independent interest:

Theorem 1.2. Let $\mathbf{j}$ denote the central involution of $\operatorname{Sp}_{2 n}(q)$. Then for any element $g \in \operatorname{Sp}_{2 n}(q)$,

$$
\omega(g) \omega(\mathbf{j} g)=\epsilon^{n} \omega\left(g^{2}\right), \quad \omega^{*}(g) \omega^{*}(\mathbf{j} g)=\epsilon^{n} \omega^{*}\left(g^{2}\right)
$$


if $q \equiv \pm 1(\bmod 8)$, and

$$
\omega(g) \omega(\mathbf{j} g)=\epsilon^{n} \omega^{*}\left(g^{2}\right), \quad \omega^{*}(g) \omega^{*}(\mathbf{j} g)=\epsilon^{n} \omega\left(g^{2}\right)
$$

if $q \equiv \pm 3(\bmod 8)$.

In the next theorem, we construct, for the first time, infinite series of examples of irreducible symmetric cubes and alternating cubes of complex representations (of finite groups of Lie type of degree $>1$ ), as well as irreducible tensor products of complex representations, with one factor being non-Weil. Note that previous examples of the latter kind [18] all involve only Weil representations. Furthermore, it was shown in [11] that, if $k \geq 4$ and $\Phi$ is a complex irreducible representation of a finite group $G$ of degree $\geq 5$ then $\operatorname{Sym}^{k}(\Phi)$ cannot be irreducible, unless $G$ is one of a few (explicitly known) groups of small order. In fact, we also exhibit examples of complex representations $\Phi$ of degree $>1$ with $\mathrm{S}_{2,1}(\Phi)$ being irreducible, where $\mathrm{S}_{2,1}(\cdot)$ is the Schur functor labeled by the partition $(2,1)$, cf. [6, §6.1]. Questions about irreducibility of tensor products and symmetric/alternating powers (or more generally, of $S_{\lambda}(\Phi)$ for Schur functors $S_{\lambda}$ labeled by partitions $\lambda$ of a small positive integer) of representations $\Phi$ of finite groups are of interest to the Aschbacher-Scott program [1], [2] of classifying maximal subgroups of finite classical groups, cf. [15], [16], [17], [19].

Theorem 1.3. Suppose that $n \geq 3$. Then the following characters

$$
\begin{array}{r}
\wedge^{3}(\xi), \operatorname{Sym}^{3}(\eta), \operatorname{Sym}^{3}(\xi)-\bar{\xi}, \wedge^{3}(\eta)-\bar{\eta}, \operatorname{Sym}^{2}(\xi) \otimes \eta \\
\wedge^{2}(\eta) \otimes \xi, \wedge^{2}(\xi) \otimes \eta-\bar{\eta}, \operatorname{Sym}^{2}(\eta) \otimes \xi-\bar{\xi}, \mathrm{S}_{2,1}(\xi), \mathrm{S}_{2,1}(\eta)
\end{array}
$$

of $\mathrm{Sp}_{2 n}(3)$ are all irreducible and pairwise distinct.

Some (but not all) of our results can also be extended to the modular case. We restrict ourselves to formulate the result in some interesting cases. Let $G=\operatorname{Sp}_{2 n}(q)$ with $q$ odd as above, and let $\mathbb{F}$ be an algebraically closed field of characteristic $\ell \nmid 2 q$. It is well known, cf. [10, Proposition 13.6], that there are irreducible $\mathbb{F} G$-modules $U$, respectively, $\tilde{U}, W$, and $\tilde{W}$, whose Brauer characters are the restriction of $\eta, \eta^{*}, \xi$, and $\xi^{*}$, respectively, to the $\ell^{\prime}$-elements of $G$. Recall [32] that if $k \geq 3$ then $q^{k}-1$ admits a primitive prime divisor $\operatorname{ppd}(q, k)$, that is, a prime divisor of $q^{k}-1$ that does not divide $\prod_{j=1}^{k-1}\left(q^{j}-1\right)$. Note that $\operatorname{ppd}(q, 2 n)$ divides $q^{n}+1$. 
Theorem 1.4. Suppose that either $\ell \nmid|G|$, or $\ell=\operatorname{ppd}(q, 2 n)$ and $n \geq 2$, or $\ell=\operatorname{ppd}(q, n)$ and $n \geq 3$ is odd, or $n=1$ and $q \equiv 1(\bmod 4)$ and $\ell \mid(q+1) / 2$, or $n=1$ and $q \equiv 3(\bmod 4)$ and $\ell \mid(q-1) / 2$. Then the following statements hold.

(i) The modules $U \otimes W, \tilde{U} \otimes \tilde{W}, \operatorname{Sym}^{2}(U), \operatorname{Sym}^{2}(\tilde{U}), \wedge^{2}(W), \wedge^{2}(\tilde{W})$ are all semisimple. Furthermore, $U \otimes W \cong \tilde{U} \otimes \tilde{W}$. If $q \equiv \pm 1(\bmod 8)$ then

$$
\operatorname{Sym}^{2}(U) \cong \operatorname{Sym}^{2}(\tilde{U}) \cong \wedge^{2}(W) \cong \wedge^{2}(\tilde{W}) .
$$

If $q \equiv \pm 3(\bmod 8)$, then

$$
\operatorname{Sym}^{2}(U) \cong \wedge^{2}(\tilde{W}), \quad \operatorname{Sym}^{2}(\tilde{U}) \cong \wedge^{2}(W)
$$

(ii) Suppose that $q=3$ and $n \geq 3$. Then the modules

$$
\wedge^{3}(W), \operatorname{Sym}^{3}(U), \operatorname{Sym}^{2}(W) \otimes U, \wedge^{2}(U) \otimes W, \mathrm{~S}_{2,1}(U), \mathrm{S}_{2,1}(W)
$$

of $\operatorname{Sp}_{2 n}(3)$ are all irreducible.

\section{Weil Character Values}

Recall that we have fixed a nontrivial linear character $\psi$ of the additive group $\left(\mathbb{F}_{q},+\right)$, and let $\psi^{*}(x)=\psi(a x)$ for some non-square element $a \in \mathbb{F}_{q}^{\times}$. Let the functions $\gamma, \gamma^{*}: \mathbb{F}_{q} \rightarrow \mathbb{C}$ be defined via

$$
\gamma(b)=q^{-1 / 2} \sum_{x \in \mathbb{F}_{q}} \psi\left(b x^{2}\right), \quad \gamma^{*}(b)=q^{-1 / 2} \sum_{x \in \mathbb{F}_{q}} \psi^{*}\left(b x^{2}\right), \quad \forall b \in \mathbb{F}_{q} .
$$

Also, let $\chi$ denote the unique linear character of order 2 of the multiplicative group $\mathbb{F}_{q}^{\times}$, and let

$$
d(x):=\operatorname{dim}_{\mathbb{F}_{q}} \operatorname{Ker}(x)
$$

for any $x \in \operatorname{End}(V)$.

A key role in our arguments is played by the following result [23, Theorem 1A, Remark 1.3]:

Theorem 2.1. There is a function $\sigma: \operatorname{Sp}_{2 n}(q) \rightarrow \mathbb{F}_{q}^{\times}$such that the following statements hold. 
(i) If $g \in \operatorname{Sp}_{2 n}(q)$ then

$$
\omega(g)=q^{(1 / 2) d\left(g-1_{V}\right)} \gamma(1)^{2 n-d\left(g-1_{V}\right)} \chi(\sigma(g)) .
$$

(ii) If $g \in \operatorname{Sp}_{2 n}(q)$ and $\operatorname{det}\left(g-1_{V}\right) \neq 0$, then $\sigma(g)=\operatorname{det}\left(g-1_{V}\right)$.

First we record the values of $\gamma(1)$ and $\gamma^{*}(1)$ :

Lemma 2.2. $\gamma^{*}(1)=-\gamma(1)$ and $\left\{\gamma(1), \gamma^{*}(1)\right\}=\left\{\epsilon^{1 / 2},-\epsilon^{1 / 2}\right\}$.

Proof. This follows, for instance, from formula (ii) in $[23, \S 2]$ and the wellknown character table of $\operatorname{Sp}_{2}(q) \cong \mathrm{SL}_{2}(q)$, which shows that $\left\{\omega(t), \omega^{*}(t)\right\}=$ $\left\{(\epsilon q)^{1 / 2},-(\epsilon q)^{1 / 2}\right\}$ for a transvection $t \in \operatorname{Sp}_{2}(q)$ when $n=1$, see e.g. $[26$, Lemma 2.6(iii)].

Fix a decomposition $V=A \oplus A^{*}$ into a direct sum of two maximal totally isotropic subspaces. Then the stabilizer of this decomposition is isomorphic to $\mathrm{GL}(A) \cong \mathrm{GL}_{n}(q)$, and the restriction of $\omega=\omega_{n}$ to $\mathrm{GL}_{n}(q)$ gives rise to the reducible Weil character

$$
\tau_{n}: g \mapsto q^{\operatorname{dim}_{\mathbb{F}_{q}} \operatorname{Ker}\left(g-1_{A}\right)}
$$

of $\operatorname{GL}(A)$ (which is just the permutation character of the action of $\operatorname{GL}(A)$ on the point set of $\left.A \cong \mathbb{F}_{q}^{n}\right)$. Similarly, one can embed $\mathrm{GU}(B) \cong \mathrm{GU}_{n}(q)$ in $\operatorname{Sp}_{2 n}(q)$ (where $B=\mathbb{F}_{q^{2}}^{n}$ is endowed with a non-degenerate Hermitian form), and then the restriction of $\omega_{n}$ to $\mathrm{GU}_{n}(q)$ gives rise to the reducible Weil character

$$
\zeta_{n}: g \mapsto(-1)^{n}(-q) \operatorname{dim}_{\mathbb{F}^{2}} \operatorname{Ker}\left(g-1_{B}\right)
$$

of $\mathrm{GU}(B)$, cf. [8].

On the other hand, $G=\operatorname{Sp}_{2 n}(q)$ can also be naturally embedded in $\mathrm{GL}_{2 n}(q)$ and $\mathrm{GU}_{2 n}(q)$, and one has the following formulae for the restriction of $\tau_{2 n}$ and $\zeta_{2 n}$ to $G$ :

Lemma 2.3. The following statements hold.

(i) For any $g \in \operatorname{Sp}_{2 n}(q), \omega^{*}(g)=(-1)^{d\left(g-1_{V}\right)} \omega(g)$.

(ii) If $q \equiv 1(\bmod 4)$, then $\left(\omega_{n}\right)^{2}=\left(\omega_{n}^{*}\right)^{2}=\left.\left(\tau_{2 n}\right)\right|_{G}$ and $\omega_{n} \omega_{n}^{*}=\left.\left(\zeta_{2 n}\right)\right|_{G}$.

(iii) If $q \equiv 3(\bmod 4)$, then $\left(\omega_{n}\right)^{2}=\left(\omega_{n}^{*}\right)^{2}=\left.\left(\zeta_{2 n}\right)\right|_{G}$ and $\omega_{n} \omega_{n}^{*}=\left.\left(\tau_{2 n}\right)\right|_{G}$. 
Proof. (i) follows from Theorem 2.1(i) and Lemma 2.2. By the same statements, we also have

$$
\omega(g)^{2}=q^{d\left(g-1_{V}\right)} \epsilon^{2 n-d\left(g-1_{V}\right)}=(\epsilon q)^{d\left(g-1_{V}\right)},
$$

and

$$
\omega(g) \omega^{*}(g)=q^{d\left(g-1_{V}\right)}(-\epsilon)^{2 n-d\left(g-1_{V}\right)}=(-\epsilon q)^{d\left(g-1_{V}\right)},
$$

whence (ii) and (iii) follow.

Note that the function $\sigma$ is multiplicative in the sense that if $V=A \oplus B$ is an orthogonal sum of two non-degenerate subspaces and $g=x y$ with $x \in \operatorname{Sp}(A)$ and $y \in \operatorname{Sp}(B)$, then $\sigma(g)=\sigma(x) \sigma(y)$, cf. [23, §4.2.1]. It follows from Theorem 2.1(i) that

$$
\omega_{n}(g)=\omega_{k}(x) \omega_{n-k}(y)
$$

if $\operatorname{dim} A=2 k$ and $\operatorname{dim} B=2 n-2 k$.

Proposition 2.4. For any $g \in \operatorname{Sp}_{2 n}(q)$, the following statements hold.

(i) $d\left(g^{2}-1_{V}\right)=d\left(g-1_{V}\right)+d\left(\mathbf{j} g-1_{V}\right)$.

(ii) $d\left(\mathbf{j} g^{2}-1_{V}\right)$ is even. In particular, $\omega\left(\mathbf{j} g^{2}\right)=\omega^{*}\left(\mathbf{j} g^{2}\right)$.

Proof. Without loss we may extend the symplectic form $(\cdot, \cdot)$ to $\tilde{V}:=$ $V \otimes_{\mathbb{F}_{q}} \overline{\mathbb{F}}_{q}$ and then replace $V$ by $\tilde{V}$ (which we then denote by $V$ again), and $\operatorname{Sp}_{2 n}\left(\mathbb{F}_{q}\right)$ by $\operatorname{Sp}_{2 n}\left(\overline{\mathbb{F}}_{q}\right)$. Let $s$ and $u$ denote the semisimple and unipotent part of $g$, and let $V_{\lambda}:=\operatorname{Ker}\left(s-\lambda \cdot 1_{V}\right)$ for any $\lambda \in \overline{\mathbb{F}}_{q}^{\times}$. Also, let $i$ and $-i$ denote the two square roots of -1 in $\overline{\mathbb{F}}_{q}$. Then

$$
V=\bigoplus_{\lambda \in \overline{\mathbb{F}}_{q}^{\times}} V_{\lambda} .
$$

(i) It suffices to show that $\operatorname{Ker}\left(g^{2}-1_{V}\right)=\operatorname{Ker}\left(g-1_{V}\right) \oplus \operatorname{Ker}\left(g+1_{V}\right)$. One inclusion is obvious. For the other inclusion, let $v \in \operatorname{Ker}\left(g^{2}-1_{V}\right)$. Then $s^{2}(v)=v$, and the decomposition (2.4) implies that $v=v_{+}+v_{-}$with $v_{+} \in V_{1}$ and $v_{-} \in V_{-1}$. As $g^{2}=s^{2} u^{2}$ fixes $v$ and stabilizes each of $V_{1}$ and $V_{-1}$, we must have that

$$
u^{2}\left(v_{+}\right)=v_{+}, \quad u^{2}\left(v_{-}\right)=v_{-} .
$$


Note that the order of the unipotent element $u$ is odd, so (2.5) implies that $u$ fixes each of $v_{+}$and $v_{-}$. Thus $v_{+} \in \operatorname{Ker}\left(g-1_{V}\right)$ and $v_{-} \in \operatorname{Ker}\left(g+1_{V}\right)$, and the statement follows.

(ii) Note that $V_{\lambda} \perp V_{\mu}$ whenever $\lambda \mu \neq 1$; in particular, $V_{ \pm i} \perp V_{\lambda}$ whenever $\lambda \neq \pm i$. It follows that $W:=V_{i} \oplus V_{-i}$ is a $g$-invariant non-degenerate subspace of $V$. Furthermore, the subspaces $V_{i}$ and $V_{-i}$ are both totally isotropic, and of the same dimension since $s$ and $s^{-1}$ are conjugate in $\operatorname{Sp}(V)$, see [28, Proposition 3.1]. Thus both $V_{ \pm i}$ are maximal totally isotropic subspaces of $W$, and we can identify $V_{-i}$ with the dual space $V_{i}^{*}$. As $u$ stabilizes each of $V_{ \pm i}$, it follows that $\operatorname{Ker}\left(\left(\left.u\right|_{V_{i}}\right)^{2}-1_{V_{i}}\right)$ and $\operatorname{Ker}\left(\left(\left.u\right|_{V_{-i}}\right)^{2}-1_{V_{-i}}\right)$ have the same dimension. Arguing as in (i), we can show that

$$
\operatorname{Ker}\left(\mathbf{j} g^{2}-1_{V}\right)=\operatorname{Ker}\left(\left(\left.u\right|_{V_{i}}\right)^{2}-1_{V_{i}}\right) \oplus \operatorname{Ker}\left(\left(\left.u\right|_{V_{-i}}\right)^{2}-1_{V_{-i}}\right) .
$$

Hence $\operatorname{Ker}\left(\mathbf{j} g^{2}-1_{V}\right)$ has even dimension, yielding the first part of the statement. The second part now follows from Lemma 2.3(i).

It suffices to prove just one of the two identities in Theorem 1.2 (in each of the two cases for $q$ ); the other identity then follows using a conjugation by some $x \in \mathrm{CSp}_{2 n}(q)$.

Proposition 2.5. Theorem 1.2 holds if $g \in \operatorname{Sp}_{2 n}(q)$ is unipotent.

Proof. (i) Since both $\omega$ and $\omega^{*}$ are multiplicative, see (2.3), it suffices to prove the statement in the case $g$ fixes no proper non-degenerate subspace of $V$. Since $g$ is unipotent, it follows from the proof of [27, Proposition 7.1] that the Jordan canonical form of $g$ on $V$ is either a single block $J_{2 k}$ of even size $2 k$, or a direct sum $t J_{k}$ of $t \geq 1$ Jordan blocks of the same odd size $k$ (in fact $t=2$, but we do not need it).

Note that $\operatorname{det}\left(\mathbf{j} g-1_{V}\right)=(-2)^{2 n}$ is a square in $\mathbb{F}_{q}^{\times}$, so by Theorem 2.1 and Lemma 2.2 we have that $\chi(\sigma(\mathbf{j} g))=1$ and

$$
\omega(\mathbf{j} g)=\epsilon^{n} .
$$

Suppose in addition that $q \equiv \pm 1(\bmod 8)$. Then 2 is a square in $\mathbb{F}_{q}^{\times}$by quadratic reciprocity. By [27, Proposition 7.1] and its proof, $g$ and $g^{2}$ are conjugate in $G=\operatorname{Sp}_{2 n}(q)$. In particular, $\omega(g)=\omega\left(g^{2}\right)$, and so we are done 
by (2.6). So it remains to consider the case $q \equiv \pm 3(\bmod 8)$, equivalently, 2 is not a square in $\mathbb{F}_{q}^{\times}$.

(ii) Consider the case $g$ acts on $V$ as $J_{2 k}$, i.e. $k=n$ and $g$ is a regular unipotent element. By [27, Lemma 7.2], there is some $x \in H \backslash \mathbf{Z}(H) G$ conjugating $g$ to $g^{2}$, where $H=\operatorname{CSp}_{2 n}(q)$. As mentioned above, $H$ fuses $\omega$ and $\omega^{*}$, and certainly $\mathbf{Z}(H) G$ fixes each of $\omega$ and $\omega^{*}$. Hence $\omega^{*}=\omega^{x}$, and SO

$$
\omega^{*}\left(g^{2}\right)=\omega^{x}\left(g^{x}\right)=\omega(g)
$$

and we are again done by (2.6).

(iii) Now we may assume that the Jordan canonical form of $g$ on $V$ is $t J_{k}$ for some odd $k$. By part (c) of the proof of [27, Proposition 7.1], $g$ and $g^{2}$ are conjugate in $G$, and so $\omega(g)=\omega\left(g^{2}\right)$ and also $d\left(g-1_{V}\right)=d\left(g^{2}-1_{V}\right)$. As $t k=2 n$, we have that $d\left(g-1_{V}\right)=t$ is even, whence $d\left(g^{2}-1_{V}\right)$ is even. The latter implies by Lemma 2.3(i) that $\omega^{*}\left(g^{2}\right)=\omega\left(g^{2}\right)$. Thus $\omega^{*}\left(g^{2}\right)=\omega(g)$, and we are done by (2.6).

Proof of Theorem 1.2. Let $s$ denote the semisimple part of $g$. Then we can decompose $V$ as an orthogonal sum $V=V^{\prime} \oplus V_{1} \oplus V_{-1}$ of $g$-invariant non-degenerate subspaces, where $s$ acts as $1_{V_{1}}$ on $V_{1},-1_{V_{-1}}$ on $V_{-1}$, and neither 1 nor -1 is an eigenvalue for $\left.s\right|_{V^{\prime}}$. Again using the multiplicativity of $\omega$ and $\omega^{*}$ in the sense of (2.3), it suffices to prove the statement in the case $g$ fixes no proper non-degenerate subspace of $V$. The latter condition on $g$ implies that exactly one of the three summands $V^{\prime}, V_{1}, V_{-1}$ is nonzero (and then equal to $V$ ).

Suppose $V=V_{1}$. Then $g$ is unipotent, and we are done by Proposition 2.5 .

Next suppose that $V=V_{-1}$. Then $\mathbf{j} g$ is unipotent and $g^{2}=(\mathbf{j} g)^{2}$, and so we are again done by applying Proposition 2.5 to $\mathbf{j} g$.

Thus we may assume now that $V=V^{\prime}$, i.e. $\operatorname{det}\left(g^{2}-1_{V}\right) \neq 0$ and $d\left(g^{2}-1_{V}\right)=0$. By Proposition 2.4(i),

$$
d\left(g-1_{V}\right)=d\left(\mathbf{j} g-1_{V}\right)=0, \quad \operatorname{det}\left(g-1_{V}\right) \neq 0, \quad \operatorname{det}\left(\mathbf{j} g-1_{V}\right) \neq 0 .
$$


It follows by Theorem 2.1 and Lemma 2.2 that

$$
\omega(g)=\epsilon^{n} \chi\left(\operatorname{det}\left(g-1_{V}\right)\right), \omega(\mathbf{j} g)=\epsilon^{n} \chi\left(\operatorname{det}\left(\mathbf{j} g-1_{V}\right)\right)=\epsilon^{n} \chi\left(\operatorname{det}\left(g+1_{V}\right)\right) .
$$

Hence

$$
\omega(g) \omega(\mathbf{j} g)=\chi\left(\operatorname{det}\left(g^{2}-1_{V}\right)\right) .
$$

On the other hand, Theorem 2.1 and Lemma 2.2 applied to $g^{2}$ yield

$$
\omega^{*}\left(g^{2}\right)=\epsilon^{n} \chi\left(\operatorname{det}\left(g^{2}-1_{V}\right)\right)=\omega\left(g^{2}\right),
$$

and the statement follows.

\section{Symmetric and Alternating Squares}

In this section, we set $\delta:=\epsilon^{n}=(-1)^{n(q-1) / 2}$. First we give a formula for the irreducible Weil characters in terms of $\omega$ and $\omega^{*}$ :

Lemma 3.1. The following statements hold for any $g \in G=\operatorname{Sp}_{2 n}(q)$ :

$$
\begin{aligned}
\xi(g)=\frac{\omega(g)+\delta \omega(\mathbf{j} g)}{2}, & \eta(g)=\frac{\omega(g)-\delta \omega(\mathbf{j} g)}{2} \\
\xi^{*}(g)=\frac{\omega^{*}(g)+\delta \omega^{*}(\mathbf{j} g)}{2}, & \eta^{*}(g)=\frac{\omega^{*}(g)-\delta \omega^{*}(\mathbf{j} g)}{2} .
\end{aligned}
$$

Proof. By [26, Lemma 2.6(i)], j acts on any $G$-module affording the character $\xi$ or $\xi^{*}$ as the multiplication by $\delta$. Similarly, $\mathbf{j}$ acts on any $G$-module affording the character $\eta$ or $\eta^{*}$ as the multiplication by $-\delta$. Hence

$$
\omega(g)=\xi(g)+\eta(g), \quad \omega(\mathbf{j} g)=\xi(\mathbf{j} g)+\eta(\mathbf{j} g)=\delta \xi(g)-\delta \eta(g),
$$

and the statements follow.

Corollary 3.2. $\xi \eta=\xi^{*} \eta^{*}$.

Proof. By Lemma 3.1, for any $g \in \operatorname{Sp}_{2 n}(q)$ we have

$$
\xi(g) \eta(g)=\frac{\omega(g)^{2}-\omega(\mathbf{j} g)^{2}}{4}, \quad \xi^{*}(g) \eta^{*}(g)=\frac{\omega^{*}(g)^{2}-\omega^{*}(\mathbf{j} g)^{2}}{4} .
$$

Since $\omega(h)^{2}=\omega^{*}(h)^{2}$ for all $h \in \operatorname{Sp}_{2 n}(q)$ by Lemma 2.3, the statement follows. 


\section{Lemma 3.3.}

(i) If $q \equiv \pm 1(\bmod 8)$ then $\operatorname{Sym}^{2}(\xi) \neq \operatorname{Sym}^{2}\left(\xi^{*}\right)$ and $\wedge^{2}(\eta) \neq \wedge^{2}\left(\eta^{*}\right)$.

(ii) If $q \equiv \pm 3(\bmod 8)$ then $\wedge^{2}(\xi) \neq \wedge^{2}\left(\xi^{*}\right)$ and $\operatorname{Sym}^{2}(\eta) \neq \operatorname{Sym}^{2}\left(\eta^{*}\right)$.

(iii) In general, $\xi \eta^{*} \neq \xi^{*} \eta$.

Proof. (i) Consider a transvection $t \in G=\operatorname{Sp}_{2 n}(q)$. As mentioned in part (i) of the proof of Proposition $2.5, q \equiv \pm 1(\bmod 8)$ implies that $t^{2}$ is conjugate to $t$ in $G$. Hence [26, Lemma 2.6(iii)] implies that

$$
\operatorname{Sym}^{2}(\xi)(t)=\frac{3+\epsilon q^{2 n-1}+4 q^{n-1} \sqrt{\epsilon q}}{8}, \operatorname{Sym}^{2}\left(\xi^{*}\right)(t)=\frac{3+\epsilon q^{2 n-1}-4 q^{n-1} \sqrt{\epsilon q}}{8} .
$$

In particular, $\operatorname{Sym}^{2}(\xi) \neq \operatorname{Sym}^{2}\left(\xi^{*}\right)$. Similarly, $\wedge^{2}(\eta) \neq \wedge^{2}\left(\eta^{*}\right)$.

(ii) We again evaluate relevant characters at a transvection $t \in G$. Since $q \equiv$ $\pm 3(\bmod 8), 2$ is not a square in $\mathbb{F}_{q}^{\times}$. Hence by the proof of $[27$, Proposition 7.1], there is some $x \in H \backslash \mathbf{Z}(H) G$ that conjugates $t$ to $t^{2}$, where $H=$ $\mathrm{CSp}_{2 n}(q)$ as usual. As mentioned above, $\eta^{x}=\eta^{*}$, hence

$$
\eta^{*}\left(t^{2}\right)=\eta^{x}\left(t^{2}\right)=\eta(t)
$$

Now using [26, Lemma 2.6(iii)] we can see that

$$
\operatorname{Sym}^{2}\left(\eta^{*}\right)=\frac{\eta^{*}(t)^{2}+\eta(t)}{2}=\frac{-1+\epsilon q^{2 n-1}+4 q^{n-1} \sqrt{\epsilon q}}{8} .
$$

Similarly, one computes that

$$
\operatorname{Sym}^{2}(\eta)=\frac{\eta(t)^{2}+\eta^{*}(t)}{2}=\frac{-1+\epsilon q^{2 n-1}-4 q^{n-1} \sqrt{\epsilon q}}{8}
$$

and so $\operatorname{Sym}^{2}(\eta) \neq \operatorname{Sym}^{2}\left(\eta^{*}\right)$. The inequality $\wedge^{2}(\xi) \neq \wedge^{2}\left(\xi^{*}\right)$ is proved in the same way.

(iii) Again we use [26, Lemma 2.6(iii)] to see

$$
\xi(t) \eta^{*}(t)=\frac{-\left(1+q^{n-1} \sqrt{\epsilon q}\right)^{2}}{4} \neq \frac{-\left(1-q^{n-1} \sqrt{\epsilon q}\right)^{2}}{4}=\xi^{*}(t) \eta(t) .
$$


Proof of Theorem 1.1. By Lemma 3.1 and Lemma 2.3(i) we have

$$
\begin{aligned}
\wedge^{2}(\xi)(g) & =\frac{\omega(g)^{2}+\omega(\mathbf{j} g)^{2}+2 \delta \omega(g) \omega(\mathbf{j} g)}{8}-\frac{\omega\left(g^{2}\right)+\delta \omega\left(\mathbf{j} g^{2}\right)}{4} \\
\wedge^{2}\left(\xi^{*}\right)(g) & =\frac{\omega(g)^{2}+\omega(\mathbf{j} g)^{2}+2 \delta \omega^{*}(g) \omega^{*}(\mathbf{j} g)}{8}-\frac{\omega^{*}\left(g^{2}\right)+\delta \omega^{*}\left(\mathbf{j} g^{2}\right)}{4} .
\end{aligned}
$$

Similarly,

$$
\begin{gathered}
\operatorname{Sym}^{2}(\eta)(g)=\frac{\omega(g)^{2}+\omega(\mathbf{j} g)^{2}-2 \delta \omega(g) \omega(\mathbf{j} g)}{8}+\frac{\omega\left(g^{2}\right)-\delta \omega\left(\mathbf{j} g^{2}\right)}{4}, \\
\operatorname{Sym}^{2}\left(\eta^{*}\right)(g)=\frac{\omega(g)^{2}+\omega(\mathbf{j} g)^{2}-2 \delta \omega^{*}(g) \omega^{*}(\mathbf{j} g)}{8}+\frac{\omega^{*}\left(g^{2}\right)-\delta \omega^{*}\left(\mathbf{j} g^{2}\right)}{4} .
\end{gathered}
$$

Also, by Proposition 2.4(ii) we have

$$
\omega\left(\mathbf{j} g^{2}\right)=\omega^{*}\left(\mathbf{j} g^{2}\right)
$$

(i) First we consider the case $q \equiv \pm 1(\bmod 8)$. By Theorem 1.2 ,

$$
\omega(g) \omega(\mathbf{j} g)=\delta \omega\left(g^{2}\right), \quad \omega^{*}(g) \omega^{*}(\mathbf{j} g)=\delta \omega^{*}\left(g^{2}\right) .
$$

Together with (3.1), (3.2), and (3.3), this implies that

$$
\wedge^{2}(\xi)=\wedge^{2}\left(\xi^{*}\right)=\operatorname{Sym}^{2}(\eta)=\operatorname{Sym}^{2}\left(\eta^{*}\right)
$$

(ii) Assume now that $q \equiv \pm 3(\bmod 8)$. By Theorem 1.2 ,

$$
\omega(g) \omega(\mathbf{j} g)=\delta \omega^{*}\left(g^{2}\right), \quad \omega^{*}(g) \omega^{*}(\mathbf{j} g)=\delta \omega\left(g^{2}\right) .
$$

Together with (3.1), (3.2), and (3.4), this implies that

$$
\wedge^{2}(\xi)=\operatorname{Sym}^{2}\left(\eta^{*}\right), \quad \wedge^{2}\left(\xi^{*}\right)=\operatorname{Sym}^{2}(\eta)
$$


We also have by Lemma 3.1 and Lemma 2.3(i) that

$$
\begin{aligned}
\operatorname{Sym}^{2}(\xi)(g) & =\frac{\omega(g)^{2}+\omega(\mathbf{j} g)^{2}+2 \delta \omega(g) \omega(\mathbf{j} g)}{8}+\frac{\omega\left(g^{2}\right)+\delta \omega\left(\mathbf{j} g^{2}\right)}{4}, \\
\operatorname{Sym}^{2}\left(\xi^{*}\right)(g) & =\frac{\omega(g)^{2}+\omega(\mathbf{j} g)^{2}+2 \delta \omega^{*}(g) \omega^{*}(\mathbf{j} g)}{8}+\frac{\omega^{*}\left(g^{2}\right)+\delta \omega^{*}\left(\mathbf{j} g^{2}\right)}{4}, \\
\wedge^{2}(\eta)(g) & =\frac{\omega(g)^{2}+\omega(\mathbf{j} g)^{2}-2 \delta \omega(g) \omega(\mathbf{j} g)}{8}-\frac{\omega\left(g^{2}\right)-\delta \omega\left(\mathbf{j} g^{2}\right)}{4} \\
\wedge^{2}\left(\eta^{*}\right)(g) & =\frac{\omega(g)^{2}+\omega(\mathbf{j} g)^{2}-2 \delta \omega^{*}(g) \omega^{*}(\mathbf{j} g)}{8}-\frac{\omega^{*}\left(g^{2}\right)-\delta \omega^{*}\left(\mathbf{j} g^{2}\right)}{4} .
\end{aligned}
$$

Using (3.3) and (3.4), we arrive at

$$
\operatorname{Sym}^{2}(\xi)=\operatorname{Sym}^{2}\left(\xi^{*}\right), \quad \wedge^{2}(\eta)=\wedge^{2}\left(\eta^{*}\right)
$$

(iii) The remaining statements in Theorem 1.1 now follow from Corollary 3.2 and Lemma 3.3.

Note that in the case $q=3,5$ formula (3.5) and Corollary 3.2 were established in [18, Proposition 5.5], but by different means, along the lines of $\S 4$. We also note that, even though special unitary groups $\mathrm{SU}_{n}(q)$ admit complex irreducible Weil characters of degree $k$ and $k+1$ for a suitable $k$, the symmetric square of the smaller-degree character does not coincide with the alternating square of another Weil character of the larger degree.

A further remark is that Theorem 1.1 establishes the existence of some isomorphisms between certain symmetric and alternating squares of complex Weil modules. It remains a question if one can construct natural isomorphisms between these pairs of complex modules in those cases.

\section{Symmetric and Alternating Cubes}

Recall the Weil character $\tau_{n}$ of $\mathrm{GL}_{n}(q)$ as defined in (2.1).

Lemma 4.1. Fix a positive integer $k$ and let

$$
s(k, n):=\left[\left.\left(\tau_{2 n}\right)^{k}\right|_{\operatorname{Sp}_{2 n}(q)}, 1_{\operatorname{Sp}_{2 n}(q)}\right]_{\operatorname{Sp}_{2 n}(q)} .
$$

Then $s(k, n)=s(k, k)$ for all $n \geq k$. 
Proof. Note that $s(k, n)$ is just the number of $G$-orbits on the set $V^{k}$ of ordered $k$-tuples $\left(v_{1}, \ldots, v_{k}\right)$, with $v_{i} \in V=\mathbb{F}_{q}^{2 n}$ and $G=\operatorname{Sp}(V)$. Fix a symplectic basis $\left(e_{1}, \ldots, e_{n}, f_{1}, \ldots, f_{n}\right)$ of $V$, i.e. $\left(e_{i}, e_{j}\right)=0=\left(f_{i}, f_{j}\right)$ and $\left(e_{i}, f_{j}\right)=\delta_{i, j}$. Also, let

$$
U_{j}:=\left\langle e_{1}, \ldots, e_{j}, f_{1}, \ldots, f_{j}\right\rangle_{\mathbb{F}_{q}}
$$

for $1 \leq j \leq k$.

(i) First we show that any $G$-orbit on $V^{k}$ intersects $\left(U_{k}\right)^{k}$, which implies that $s(k, n) \leq s(k, k)$. In fact, we will prove by induction on $1 \leq j \leq k$ that the $G$-orbit of any $\alpha=\left(v_{1}, \ldots, v_{j}\right) \in V^{j}$ intersects $\left(U_{j}\right)^{j}$.

For the induction base $j=1$, we can certainly find $g \in G$ such that $g\left(v_{1}\right)=e_{1} \in U_{1}$.

For the induction step $j \geq 2$, we may assume that there is some $g \in G$ such that $g\left(v_{i}\right) \in U_{j-1}$ for all $1 \leq i \leq j-1$. Now write $g\left(v_{j}\right)=u+w$ with

$$
u \in U_{j-1}, \quad w \in W:=\left\langle e_{j}, \ldots, e_{n}, f_{j}, \ldots, f_{n}\right\rangle_{\mathbb{F}_{q}} .
$$

Then we can find $h \in \operatorname{Sp}(W) \leq G$ such that $h$ acts trivially on $U_{j-1}$ and $h(w)=e_{j}$, and observe that

$$
h g(\alpha)=\left(g\left(v_{1}\right), \ldots, g\left(v_{j-1}\right), u+e_{j}\right) \in U_{j}^{j} .
$$

(ii) Conversely, suppose that $\left(u_{1}, \ldots, u_{k}\right),\left(v_{1}, \ldots, v_{k}\right) \in U^{k}$ belong to the same $G$-orbit, i.e. there is some $g \in G$ such that $g\left(u_{i}\right)=v_{i}$ for all $i$. Then $\left.g\right|_{M}$ is an isometry between the subspaces $M:=\left\langle u_{1}, \ldots, u_{k}\right\rangle_{\mathbb{F}_{q}}$ and $\left\langle v_{1}, \ldots, v_{k}\right\rangle_{\mathbb{F}_{q}}$ of $U_{k}$. By Witt's lemma, $\left.g\right|_{M}$ extends to some $h \in \operatorname{Sp}\left(U_{k}\right)$, whence $\left(u_{1}, \ldots, u_{k}\right)$ and $\left(v_{1}, \ldots, v_{k}\right)$ belong to the same $\operatorname{Sp}\left(U_{k}\right)$-orbit. Thus $s(k, k) \leq s(k, n)$.

Corollary 4.2. For $G=\mathrm{Sp}_{2 n}(3)$ and $n \geq 3$,

$$
\left[\omega^{3}, \omega^{3}\right]_{G}=80 .
$$

Proof. By Lemma 2.3(iii),

$$
\left[\omega^{3}, \omega^{3}\right]_{G}=\left[(\omega \bar{\omega})^{3}, 1_{G}\right]_{G}=\left[\left.\left(\tau_{6}\right)^{3}\right|_{G}, 1_{G}\right]_{G}=s(3, n) .
$$


Direct computation using [7] shows that $s(3,3)=80$, and so we are done by Lemma 4.1.

Proof of Theorem 1.3. From now on, let $G=\operatorname{Sp}_{2 n}(3)$ with $n \geq 3$. By [10, Lemma 13.4],

$$
\omega^{*}=\bar{\omega}, \quad \xi^{*}=\bar{\xi}, \quad \eta^{*}=\bar{\eta} .
$$

(i) First we note by Theorem 1.1 and [18, Proposition 5.4] that the characters

$$
\begin{array}{r}
\operatorname{Sym}^{2}(\xi)=\operatorname{Sym}^{2}(\bar{\xi}), \wedge^{2}(\eta)=\wedge^{2}(\bar{\eta}), \xi \eta=\bar{\xi} \bar{\eta} \\
\operatorname{Sym}^{2}(\eta), \operatorname{Sym}^{2}(\bar{\eta}), \wedge^{2}(\xi), \wedge^{2}(\bar{\xi}), \xi \bar{\eta}, \bar{\xi} \eta
\end{array}
$$

are all irreducible, but $\wedge^{2}(\xi) \neq \wedge^{2}(\bar{\xi})$. It follows that

$$
\begin{aligned}
{\left[\operatorname{Sym}^{2}(\xi) \otimes \xi, \bar{\xi}\right]_{G} } & =\left[\operatorname{Sym}^{2}(\xi), \operatorname{Sym}^{2}(\bar{\xi})+\wedge^{2}(\bar{\xi})\right]_{G}=1 \\
{\left[\wedge^{2}(\xi) \otimes \xi, \bar{\xi}\right]_{G} } & =\left[\wedge^{2}(\xi), \operatorname{Sym}^{2}(\bar{\xi})+\wedge^{2}(\bar{\xi})\right]_{G}=0 .
\end{aligned}
$$

On the other hand,

$$
\operatorname{Sym}^{2}(\xi) \otimes \xi=\operatorname{Sym}^{3}(\xi)+\mathrm{S}_{2,1}(\xi), \quad \wedge^{2}(\xi) \otimes \xi=\wedge^{3}(\xi)+\mathrm{S}_{2,1}(\xi)
$$

where $S_{2,1}(\cdot)$ is the Schur functor labeled by the partition $(2,1)$ of 3 , see $[6$, (6.8), (6.9)]. Hence, (4.2) implies that $\left[\mathrm{S}_{2,1}(\xi), \bar{\xi}\right]_{G}=0$ and

$$
\left[\operatorname{Sym}^{3}(\xi), \bar{\xi}\right]_{G}=1
$$

in particular, $\operatorname{Sym}^{3}(\xi)$ is not irreducible. Similarly,

$$
\begin{aligned}
{\left[\operatorname{Sym}^{2}(\eta) \otimes \eta, \bar{\eta}\right]_{G} } & =\left[\operatorname{Sym}^{2}(\eta), \operatorname{Sym}^{2}(\bar{\eta})+\wedge^{2}(\bar{\eta})\right]_{G}=0, \\
{\left[\wedge^{2}(\eta) \otimes \eta, \bar{\eta}\right]_{G} } & =\left[\wedge^{2}(\eta), \operatorname{Sym}^{2}(\bar{\eta})+\wedge^{2}(\bar{\eta})\right]_{G}=1,
\end{aligned}
$$

implying that

$$
\left[\wedge^{3}(\eta), \bar{\eta}\right]_{G}=1
$$

In particular, $\wedge^{3}(\eta)$ is not irreducible. Also, by Theorem 1.1(iii) we have

$$
\begin{aligned}
& {\left[\wedge^{2}(\xi) \otimes \eta, \bar{\eta}\right]_{G}=\left[\operatorname{Sym}^{2}(\bar{\eta}) \otimes \eta, \bar{\eta}\right]_{G}=\left[\operatorname{Sym}^{2}(\bar{\eta}), \operatorname{Sym}^{2}(\bar{\eta})+\wedge^{2}(\bar{\eta})\right]_{G}=1} \\
& {\left[\operatorname{Sym}^{2}(\eta) \otimes \xi, \bar{\xi}\right]_{G}=\left[\wedge^{2}(\bar{\xi}) \otimes \xi, \bar{\xi}\right]_{G}=\left[\wedge^{2}(\bar{\xi}), \operatorname{Sym}^{2}(\bar{\xi})+\wedge^{2}(\bar{\xi})\right]_{G}=1 .}
\end{aligned}
$$


Now using (4.3), (4.4), (4.5), and (4.6), we can decompose

$$
\begin{aligned}
\omega^{3}= & (\xi+\eta)^{3}=\xi^{3}+\eta^{3}+3 \xi^{2} \otimes \eta+3 \eta^{2} \otimes \xi \\
= & \operatorname{Sym}^{3}(\xi)+\wedge^{3}(\xi)+2 \mathrm{~S}_{2,1}(\xi)+\operatorname{Sym}^{3}(\eta)+\wedge^{3}(\eta)+2 \mathrm{~S}_{2,1}(\eta) \\
& +3 \operatorname{Sym}^{2}(\xi) \otimes \eta+3 \wedge^{2}(\xi) \otimes \eta+3 \operatorname{Sym}^{2}(\eta) \otimes \xi+3 \wedge^{2}(\eta) \otimes \xi \\
= & \left.\operatorname{Sym}^{3}(\xi)-\bar{\xi}\right)+\wedge^{3}(\xi)+2 \mathrm{~S}_{2,1}(\xi)+\operatorname{Sym}^{3}(\eta)+\left(\wedge^{3}(\eta)-\bar{\eta}\right)+2 \mathrm{~S}_{2,1}(\eta) \\
& +3 \operatorname{Sym}^{2}(\xi) \otimes \eta+3\left(\wedge^{2}(\xi) \otimes \eta-\bar{\eta}\right)+3\left(\operatorname{Sym}^{2}(\eta) \otimes \xi-\bar{\xi}\right) \\
& +3 \wedge^{2}(\eta) \otimes \xi+4 \bar{\xi}+4 \bar{\eta}
\end{aligned}
$$

into a sum of true characters of $G$. This decomposition implies in particular that $\left[\omega^{3}, \omega^{3}\right]_{G} \geq 80$. On the other hand, Corollary 4.2 states that $\left[\omega^{3}, \omega^{3}\right]_{G}=$ 80. It follows that the summands in the last expression given in (4.7) for $\omega^{3}$ must be all irreducible and pairwise distinct.

\section{The Modular Case}

In this section, we prove Theorem 1.4. The statements are obvious if $\ell \nmid|G|$, so we will assume that $\ell|| G \mid$. Now the assumption on $\ell$ implies that $\ell \mid\left(q^{2 n}-1\right)$, and that any irreducible character $\theta$ of $G$ has $\ell$-defect 0 if $\ell \mid\left(q^{n}-1\right)$ but $\left(q^{n}-1\right) /(q-1) \mid \theta(1)$, or if $\ell \mid\left(q^{n}+1\right)$ but $\left(q^{n}+1\right) / 2 \mid \theta(1)$.

(a) Now, in the case of Theorem 1.4(ii), note by Theorem 1.3 that the Brauer character $\varphi$ of any module in the list is the reduction modulo $\ell$ of some irreducible character $\theta \in \operatorname{Irr}(G)$ of degree divisible by $\left(q^{2 n}-1\right) / 4$. By the aforementioned remark, $\theta$ has $\ell$-defect 0 , and so $\varphi$ is irreducible as claimed.

(b) We will now work on the proof of Theorem 1.4(i). As mentioned in $[18, \S 5]$, the restriction of the character $\tau_{2 n}$ in Lemma 2.3 to $G$ is the sum of twice the trivial character, an irreducible character $\alpha_{n}$ of degree $\left(q^{n}-1\right)\left(q^{n}+q\right) / 2(q-1)$ (of multiplicity 1$)$, an irreducible character $\beta_{n}$ of degree $\left(q^{n}+1\right)\left(q^{n}-q\right) / 2(q-1)$ (of multiplicity 1 ) if $n>1$, two irreducible characters of degree $\left(q^{2 n}-1\right) / 2(q-1)$ (each of multiplicity $1)$, and $(q-3) / 2$ irreducible characters of degree $\left(q^{2 n}-1\right) /(q-1)$ (each of multiplicity 2).

Similarly, the restriction of the character $\zeta_{2 n}$ in Lemma 2.3 to $G$ is the sum of an irreducible character $\gamma_{n}$ of degree $\left(q^{n}+1\right)\left(q^{n}+q\right) / 2(q+1)$ 
generally those cases where the centralizer is finite or small-dimensional. Two of these cases are resolved in this paper. We now give some details about the unresolved cases from [14].

We first introduce a definition and then discuss the problem cases. An organizing principle in much of this work is the notion of a fusion pattern, defined as in $[15,1.2]$.

Definition 1. A fusion pattern from the group $M$ to the group $N$ is a function $f$ from the set of conjugacy classes of $M$ to the set of conjugacy classes of $N$ such that

1. if $\kappa$ is a class in $M$ of order $n$ then $f(\kappa)$ is a class in $N$ of elements of order $n$.

2. $f$ commutes with power maps, that is, if $\kappa^{(m)}$ denotes the conjugacy class of all $m$ th powers of elements of $\kappa$, a conjugacy class in $M$, then $f\left(\kappa^{(m)}\right)=f(\kappa)^{(m)}$ for every class $\kappa$ in $M$ and all integers $m$.

There is a discussion of how we denote fusion patterns as well as a numbering system for them in the material following [14, Definition 2.3]. We have kept the numbering systems consistent throughout the progression $G_{2}<F_{4}<3 E_{6}<2 E_{7}<E_{8}$ so an $A l t_{6}$ group with Fusion Pattern 260 in $F_{4}$ would also have Fusion Pattern 260 in $3 E_{6}, 2 E_{7}$ and $E_{8}$.

Conjugacy results are summarized in [14, Table 1], and are included (with improvements from this paper) in Table 1 of this paper. Throughout this paper, we will use Atlas [8] notation or a slight variation of it for characters. When there are two characters of the same degree, we distinguish them by using subscripts $a, b, \ldots$, etc.

The two cases which are resolved in this paper are $A l t_{10}$ in $E_{8}$ and 2 Alt $_{10}$ with Fusion Pattern 1 in $E_{8}$. The respective adjoint characters are $9+35+36+84^{2}$ and $36+84+64_{a}+64_{b}$. In the case of $A l t_{10}$ in $E_{8}$, Frey was able in [14] to construct instances of an $A l t_{10}$ subgroup with this fusion pattern in both the $3 A_{8}$ and $2 D_{8}$ subgroups, but was unable to determine whether those two instances were conjugate to each other, and further, because the centralizer is 0-dimensional, was unable to rule out the possibility of Lie primitive instances of this group. The resolution of this case is given in Section 5 . 
In the case of $2 A l t_{10}$ in $E_{8}$, Frey was able in [14] to construct an instance of this group with Fusion Pattern 1 in the $2 D_{8}$ subgroup, (since the central involution of this $2 A l t_{10}$ subgroup comes from the $2 \mathrm{~B}$ conjugacy class), but was unable to determine if the class of such groups is unique in the $2 D_{8}$ subgroup, which is necessary to show that the class is unique in $E_{8}$. The resolution of this case is given in Section 4.

Finally, we list the remaining unresolved cases, and the issues for each of them.

$\underline{F_{4}}$

$A l t_{6}$ : In $F_{4}$, there is an $A l t_{6}$ subgroup with Fusion Pattern 260 which has 0-dimensional centralizer. Cohen and Wales were able to construct such a subgroup in a subgroup of $F_{4}$ of type $A_{2} A_{2}$ in [7], but were unable to show that there were no Lie primitive instances of subgroups with this fusion pattern. The 26-dimensional characters for this case are the sister characters $8_{a}+9^{2}$ and $8_{b}+9^{2}$, while the adjoint characters, are $8_{a}+8_{b}^{3}+10^{2}$ and its sister character $8_{a}^{3}+8_{b}+10^{2}$.

$\underline{3 E_{6}}:$

$A l t_{6}$ : In $3 E_{6}$, there is an $A l t_{6}$ subgroup with Fusion Pattern 252 which has 0-dimensional centralizer. In [7, 6.14], Cohen and Wales were able to construct such a subgroup in $3 E_{6}$ via a $C_{4}$ subgroup or an irreducible $A_{2}$ subgroup. There is also the possibility of Lie primitive embeddings. The 27-dimensional character for this case is $5_{a}+5_{b}+8_{a}+9_{a}$.

$\underline{2 E_{7}}:$

$A l t_{6}$ : In $2 E_{7}$ there is an $A l t_{6}$ subgroup with Fusion Pattern 244 which has 0-dimensional centralizer. There is an instance of an $A_{l} t_{6}$ subgroup with this fusion pattern in the $4 A_{7}$ subgroup. There is also an instance in a $3 A_{2} A_{5}$ subgroup of $2 E_{7}$. It is unknown whether or not these two instances are conjugate to each other. It is also possible that there are Lie primitive subgroups with this fusion pattern. The characters for this case are $8_{a}^{2}+10^{4}$ and $5_{a}^{3}+5_{b}^{3}+8_{a}^{4}+8_{b}^{3}+9^{3}+10^{2}$. 
(of multiplicity 1), an irreducible character $\delta_{n}$ of degree $\left(q^{n}-1\right)\left(q^{n}-\right.$ $q) / 2(q+1)$ (of multiplicity 1 ) if $n>1$, two irreducible characters of degree $\left(q^{2 n}-1\right) / 2(q+1)$ (each of multiplicity 1$)$, and $(q-1) / 2$ irreducible characters of degree $\left(q^{2 n}-1\right) /(q+1)$ (each of multiplicity 2$)$.

(c) Here we consider the case where $q \equiv 1(\bmod 4)$ and moreover $\ell \mid(q+1)$ if $n=1$. Set $e:=\left(q^{2 n}-1\right) / 2(q-1)$. By Theorem 1.1(i) and Lemma 2.3(ii), $\xi \eta=\xi^{*} \eta^{*}$ and $\left.\left(\tau_{2 n}\right)\right|_{G}-2 \xi \eta$ is a true character. In particular, every irreducible constituent of $\xi \eta$ must occur in $\left.\left(\tau_{2 n}\right)\right|_{G}$ with multiplicity at least 2. Clearly, $1_{G}$ is not an irreducible constituent of $\xi \eta$. It follows from the remarks in (b) that every irreducible constituent in $\xi \eta$ has degree $2 e$, whence it has $\ell$-defect 0 (and occurs with multiplicity 1 ). Hence the module $U \otimes W$ is semisimple, and similarly for $\tilde{U} \otimes \tilde{W}$. As these two modules have the same Brauer character, they are isomorphic.

It is well known, cf. [10, Corollary 13.7], that $\xi$ has Frobenius-Schur indicator +1 and $\eta$ has Frobenius-Schur indicator -1 . Thus $1_{G}$ is neither an irreducible constituent of $\wedge^{2}(\xi)$, nor of $\operatorname{Sym}^{2}(\eta)$. Next, the common degree of $\wedge^{2}(\xi)$ and $\operatorname{Sym}^{2}(\eta)$ is divisible by $e$. On the other hand, none of the integers $\alpha_{n}(1), \beta_{n}(1)$, and $\alpha_{n}(1)+\beta_{n}(1)$ is divisible by $e$. It follows from the remarks in (b) that every irreducible constituent in $\wedge^{2}(\xi)$ and in $\operatorname{Sym}^{2}(\eta)$ has degree divisible by $e$, whence they all have $\ell$-defect 0 . Hence the modules $\operatorname{Sym}^{2}(U)$ and $\wedge^{2}(W)$ are semisimple, and similarly for $\operatorname{Sym}^{2}(\tilde{U})$ and $\wedge^{2}(\tilde{W})$. In particular, if any two of these four modules have the same Brauer character, then they are also isomorphic. Hence we are done by Theorem 1.1.

(d) Here we consider the case where $q \equiv 3(\bmod 4)$ and moreover $\ell \mid(q-1)$ if $n=1$. Set $f:=\left(q^{2 n}-1\right) / 2(q+1)$. By Theorem 1.1(i) and Lemma 2.3(iii), $\xi \eta=\xi^{*} \eta^{*}$ and $\left.\left(\zeta_{2 n}\right)\right|_{G}-2 \xi \eta$ is a true character. In particular, every irreducible constituent of $\xi \eta$ must occur in $\left.\left(\zeta_{2 n}\right)\right|_{G}$ with multiplicity at least 2. It follows from the remarks in (b) that every irreducible constituent in $\xi \eta$ has degree $2 f$, whence it has $\ell$-defect 0 (and occurs with multiplicity 1). Hence the module $U \otimes W$ is semisimple, and similarly for $\tilde{U} \otimes \tilde{W}$. As these two modules have the same Brauer character, they are isomorphic.

As above, the common degree of $\wedge^{2}(\xi)$ and $\operatorname{Sym}^{2}(\eta)$ is divisible by $f$. On the other hand, none of the integers $\gamma_{n}(1), \delta_{n}(1)$, and $\gamma_{n}(1)+\delta_{n}(1)$ is divisible by $f$. It follows from the remarks in (b) that every irreducible constituent in 
$\wedge^{2}(\xi)$ and in $\operatorname{Sym}^{2}(\eta)$ has degree divisible by $f$, whence they all have $\ell$-defect 0 . Hence the modules $\operatorname{Sym}^{2}(U)$ and $\wedge^{2}(W)$ are semisimple, and similarly for $\operatorname{Sym}^{2}(\tilde{U})$ and $\wedge^{2}(\tilde{W})$. In particular, if any two of these four modules have the same Brauer character, then they are also isomorphic. Hence we are again done by Theorem 1.1, and the proof of Theorem 1.4 is completed.

\section{References}

1. M. Aschbacher, On the maximal subgroups of the finite classical groups, Invent. Math. 76 (1984), 469-514.

2. M. Aschbacher and L. Scott, Maximal subgroups of finite groups, J. Algebra, 92 (1985), 44-80.

3. B. Bolt, T. G. Room and G. E. Wall, On the Clifford collineation, transform and similarity groups. I, J. Austral. Math. Soc., 2 (1961-62), 60-79.

4. N. Dummigan, Symplectic group lattices as Mordell-Weil sublattices, J. Number Theory, 61 (1996), 365-387.

5. N. Dummigan and Pham Huu Tiep, Lower bounds for the minima of certain symplectic and unitary group lattices, Amer. J. Math., 121 (1999), 889-918.

6. W. Fulton and J. Harris, 'Representation Theory', Springer-Verlag, New York, 1991.

7. The GAP group, 'GAP - groups, algorithms, and programming', Version 4.4, 2004, http://www.gap-system.org.

8. P. Gérardin, Weil representations associated to finite fields, J. Algebra, 46 (1977), 54-101.

9. R. Gow, Even unimodular lattices associated with the Weil representation of finite symplectic group, J. Algebra, 122 (1989), 510-519.

10. B. H. Gross, Group representations and lattices, J. Amer. Math. Soc., 3 (1990), 929960.

11. R. M. Guralnick and Pham Huu Tiep, Symmetric powers and a conjecture of Kollár and Larsen, Invent. Math., 174 (2008), 505-554.

12. R. E. Howe, On the characters of Weil's representations, Trans. Amer. Math. Soc., 177 (1973), 287-298.

13. I. M. Isaacs, Characters of solvable and symplectic groups, Amer. J. Math., 95 (1973), 594-635.

14. N. Katz, Rigid local systems on $\mathbb{A}^{1}$ with finite monodromy, (preprint).

15. K. Magaard and G. Malle, Irreducibility of alternating and symmetric squares, Manuscripta Math., 95 (1998), 169-180.

16. K. Magaard, G. Malle, and Pham Huu Tiep, Irreducibility of tensor squares, symmetric squares and alternating squares, Pacific J. Math., 202 (2002), 379-427. 
17. K. Magaard, G. Röhrle, and D. Testerman, On the irreducibility of symmetrizations of cross-characteristic representations of finite classical groups, J. Pure Appl. Alg., 217 (2013), 1427-1446.

18. K. Magaard and Pham Huu Tiep, Irreducible tensor products of representations of quasi-simple finite groups of Lie type, in: 'Modular Representation Theory of Finite Groups', M. J. Collins, B. J. Parshall, L. L. Scott, eds., Walter de Gruyter, Berlin et al, 2001, 239-262.

19. G. Malle, Almost irreducible tensor squares, Comm. Algebra, 27 (1999), 1033-1051.

20. R. Scharlau and Pham Huu Tiep, Symplectic groups, symplectic spreads, codes, and unimodular lattices, J. Algebra, 194 (1997), 113-156.

21. R. Scharlau and Pham Huu Tiep, Symplectic group lattices, Trans. Amer. Math. Soc., 351 (1999), 2101-2139.

22. G. M. Seitz, Some representations of classical groups, J. London Math. Soc., 10 (1975), 115-120.

23. T. Thomas, The character of the Weil representation, J. Lond. Math. Soc., 77 (2008), 221-239.

24. Pham Huu Tiep, Globally irreducible representations of finite groups and integral lattices, Geom. Dedicata, 64 (1997), 85-123.

25. Pham Huu Tiep, Weil representations as globally irreducible representations, Math. Nachr., 184 (1997), 313-327.

26. Pham Huu Tiep and A. E. Zalesskii, Some characterizations of the Weil representations of the symplectic and unitary groups, J. Algebra, 192 (1997), 130-165.

27. Pham Huu Tiep and A. E. Zalesskii, Unipotent elements of finite groups of Lie type and realization fields of their complex representations, J. Algebra, 271 (2004), 327-390.

28. Pham Huu Tiep and A. E. Zalesskii, Real conjugacy classes in algebraic groups and finite groups of Lie type, J. Group Theory, 8 (2005), 291-315.

29. H. N. Ward, Representations of symplectic groups, J. Algebra, 20 (1972), 182-195.

30. A. Weil, Sur certains groupes d'opérateurs unitaires, Acta Math., 111 (1964), 143-211.

31. A. E. Zalesskii, Eigenvalues of matrices of complex representations of finite groups of Lie type, Lecture Note Math. no. 1352, Springer, Berlin et al, 1988, 206-218.

32. K. Zsigmondy, Zur Theorie der Potenzreste, Monatsh. Math. Phys., 3 (1892), 265-284. 